\title{
FIXED POINT AND WEAK COMMUTING MAPPING
}

\section{Yogita R Sharma}

\begin{abstract}
In this paper we prove some new fixed point theorems for weak commuting mapping on complete metric space. Our results generalize several corresponding relations in metric space of weak commuting mapping.
\end{abstract}

Keywords: Fixed point, weak commuting mapping, and metric space.

AMS Classification No (2000): 47H10, 54H25

\section{INTRODUCTION}

In 1922, Banach [1] introduced very important concept of contraction mapping, which is known as a Banach contraction principle which states that "every contraction mapping of a complete metric space into itself has a unique fixed point".

Ciric [2] gave a generalization of Banach contraction principle as:

Let $S$ be a mapping of complete metric space $(X, d)$ into itself, there exists an $\alpha \in(0,1)$ such that

(1). $d(S x, S y)$

$$
\leq \alpha \max \left\{\begin{array}{l}
d(x, y), d(x, S x), d(y, S y), \\
d(x, S y), d(y, S x)
\end{array}\right\}
$$

For all $x, y \in X$, then $\mathrm{S}$ has a unique fixed point.

In 1976, Jungck [4] investigated an interdependence between commuting mappings and fixed points, proved the followings: Let $\mathrm{T}$ be a continuous mapping of a complete metric space $(X, d)$ into itself, then $\mathrm{T}$ has a fixed point in $\mathrm{X}$, if and only if there exists an $\alpha \in(0,1)$ and a mapping $S: X \rightarrow X$ which commutes with $\mathrm{T}$ and satisfies

(2). $S(X) \subset T(X)$ and $d(S x, S y) \leq \alpha d(T x, T y)$

For all $x, y \in X$. Indeed $\mathrm{S}$ and $\mathrm{T}$ have a unique common fixed point if and only if (.2) holds for some $\alpha \in(0,1)$.

Singh [9] further generalized the above result and proved the followings:
Let two continuous and commuting mappings $\mathrm{S}$ and $\mathrm{T}$ from a complete metric space $(X, d)$ into itself satisfying the following conditions:

(3). $S(X) \subset T(X)$

$$
\begin{aligned}
d(S x, S y) \leq a[ & d(T x, S x)+d(T y, S y)] \\
& +b[d(T x, S y)+d(T y, S x)]+d(T x, T y)
\end{aligned}
$$

For all $x, y \in X$, where $a, b, c$ are non-negative real numbers satisfying $0 \leq 2 a+2 b+c<1$, then $\mathrm{S}$ and $\mathrm{T}$ have a unique common fixed point.

Ranganathan [7] has further generalized the result of Jungck [4] which gives criteria for the existence of a fixed point as follows:

Let $\mathrm{T}$ be a continuous mapping of a complete metric space $(X, d)$ into itself. Then $\mathrm{T}$ has a fixed point in $\mathrm{X}$, if and only if there exists a real number $\alpha \in(0,1)$ and a mapping $S: X \rightarrow X$ which commutes with satisfying:

(4). $S(X) \subset T(X)$

and

$d(S x, S y)$

$\leq \alpha \max \{d(T x, S x), d(T y, S y), d(T x, S y), d(T y, S x), d(T x, T y)\}$

For all $x, y \in X$. Indeed commuting mapping $\mathrm{S}$ and $\mathrm{T}$ have a unique common fixed point.

Fisher [3] proved following theorem for two commuting mappings $\mathrm{S}$ and $\mathrm{T}$. 
Theorem 1 If $\mathrm{S}$ is a mapping and $\mathrm{T}$ is a continuous mapping of the complete metric space $\mathrm{X}$ into itself and satisfying the inequality.

(5). $d(S T, T S y) \leq k[d(T x, T S y)+d(S y, S T x)]$,

For all $x, y \in X$, where $0 \leq k \leq 1 / 2$, then $\mathrm{S}$ and $\mathrm{T}$ have a unique common fixed point.

Fisher further extended his theorem and proved a common fixed point of commuting mappings $\mathrm{S}$ and $\mathrm{T}$.

Theorem 2 If $\mathrm{S}$ is a mapping and $\mathrm{T}$ is a continuous mapping of the complete metric space $\mathrm{X}$ into itself and satisfying the inequality.

(6). $d(S T, T S y) \leq k[d(T x, S T x)+d(S y, T S y)]$,

for all $x, y \in X$, where $0 \leq k \leq 1 / 2$, then $\mathrm{S}$ and $\mathrm{T}$ have a unique common fixed point.

\section{WEAK** COMMUTING MAPPINGS AND COMMON FIXED POINT}

In this section Weak $^{* *}$ commuting mappings and unique common fixed point theorem in metric space is established. First we give the following definitions:

Definition 1 According to Sessa [8] two self maps S and T defined on metric space $(X, d)$ are said to be weakly commuting maps if and only if

$d(S T x, T S x) \leq d(S x, T x)$,

For all $x \in X$.

Definition 2 wo self mappings $\mathrm{S}$ and $\mathrm{T}$ of metric space $(X, d)$ are said to be weak** commuted, if $S(X) \subset T(X)$ and for any $x \in X$,

$$
\begin{aligned}
d\left(S^{2} T^{2} x, T^{2} S^{2} x\right) & \leq d\left(S^{2} T x, T S^{2} x\right) \\
& \leq d\left(S T^{2} x, T^{2} S x\right) \leq d(S T x, T S x) \leq d\left(S^{2} x, T^{2} x\right)
\end{aligned}
$$

Definition3 A map $S: X \rightarrow X, X$ being a metric space, is called an idempotent, if $S^{2}=S$.

Example1. Let $X=[0,1]$ with Euclidean metric space and define $S$ and $\mathrm{T}$ by $S x=\frac{x}{x+3}, T x=\frac{x}{3}$ for all $x \in X$, then $\quad[0,1] \subset[0,9 / 10] \quad$ where $\quad S x=[0,1] \quad$ and $T x=[0,9 / 10]$

$$
\begin{aligned}
& d\left(S^{2} T^{2} x, T^{2} S^{2} x\right)=\frac{x}{4 x+81}-\frac{x}{36 x+81} \\
& =\frac{32 x^{2}}{(4 x+81)(36 x+81)} \\
& \leq \frac{8 x^{2}}{(4 x+27)(12 x+27)} \\
& =\frac{x}{4 x+27}-\frac{x}{12 x+27} \\
& =d\left(S^{2} T x, T S^{2} x\right) .
\end{aligned}
$$

Implies that $d\left(S^{2} T^{2} x, T^{2} S^{2} x\right) \leq d\left(S^{2} T x, T S^{2} x\right)$

$$
\begin{aligned}
d\left(S^{2} T x, T S^{2} x\right)= & \frac{x}{4 x+27}-\frac{x}{12 x+27} \\
& =\frac{8 x^{2}}{(4 x+27)(12 x+27)} \\
& \leq \frac{8 x^{2}}{(x+27)(9 x+27)} \\
& =\frac{x}{x+27}-\frac{x}{9 x+27} \\
& =d\left(S T^{2} x, T^{2} S x\right)
\end{aligned}
$$

$$
d\left(S^{2} T x, T S^{2} x\right) \leq d\left(S T^{2} x, T^{2} S x\right)
$$

$$
\begin{aligned}
d\left(S T^{2} x, T^{2} S x\right) & =\frac{x}{x+27}-\frac{x}{9 x+27} \\
& =\frac{8 x^{2}}{(x+27)(9 x+27)} \\
& \leq \frac{2 x^{2}}{(x+9)(3 x+9)} \\
& =\frac{x}{x+9}-\frac{x}{3 x+9} \\
& =d(S T x, T S x)
\end{aligned}
$$

$$
d\left(S T^{2} x, T^{2} S x\right) \leq d(S T x, T S x)
$$

$$
\begin{aligned}
& d(S T x, T S x)=\frac{x}{x+9}-\frac{x}{3 x+9} \\
& =\frac{2 x^{2}}{(x+9)(3 x+9)} \\
& \leq \frac{4 x^{2}}{9(4 x+9)} \\
& =\frac{x}{9}-\frac{x}{4 x+9} \\
& =d\left(T^{2} x, S^{2} x\right)
\end{aligned}
$$


$d(S T x, T S x) \leq d\left(T^{2} x, S^{2} x\right)$.

Using $[0,1]$ for $x \in X$, we conclude that definition (2) as follows:

$$
\begin{aligned}
d\left(S^{2} T^{2} x, T^{2} S^{2} x\right) & \leq d\left(S^{2} T x, T S^{2} x\right) \\
& \leq d\left(S T^{2} x, T^{2} S x\right) \leq d(S T x, T S x) \leq d\left(S^{2} x, T^{2} x\right)
\end{aligned}
$$

for any $x \in X$.

We further generalized the result of Fisher [3], Pathak [6], Lohani and Badshah [5] by using another type of rational expression.

Theorem1. If $\mathrm{S}$ is a mapping and $\mathrm{T}$ is a continuous mapping of complete metric space $\{S, T\}$ is weak commuting pair and the following condition:

(7).

$$
\begin{aligned}
& d\left(S^{2} T^{2} x, T^{2} S^{2} y\right) \leq \alpha \frac{\left[d\left(T^{2} x, S^{2} T^{2} x\right)\right]^{3}+\left[d\left(S^{2} y, T^{2} S^{2} y\right)\right]^{3}}{\left[d\left(T^{2} x, S^{2} T^{2} x\right)\right]^{2}+\left[d\left(S^{2} y, T^{2} S^{2} y\right)\right]^{2}} \\
& +\beta d\left(T^{2} x, S^{2} y\right),
\end{aligned}
$$

for all $\mathrm{x}$, $\mathrm{y}$ in $\mathrm{X}$, where $\alpha, \beta \geq 0$ with $2 \alpha+\beta<1$, then $\mathrm{S}$ and $\mathrm{T}$ have a unique common fixed point.

Proof Let $\mathrm{x}$ be an arbitrary point $\mathrm{X}$. Define

$\left(S^{2} T^{2}\right)^{n} x=x_{2 n}$ or $T^{2}\left(S^{2} T^{2}\right)^{n} x=x_{2 n+1}$,

where $n=0,1,2,3, \ldots$, by contractive condition (7),

$$
\begin{aligned}
& d\left(x_{2 n}, x_{2 n+1}\right)=d\left(\left(S^{2} T^{2}\right)^{n} x, T^{2}\left(S^{2} T^{2}\right)^{n} x\right) \\
& =d\left(S^{2} T^{2}\left(S^{2} T^{2}\right)^{n-1} x, T^{2} S^{2}\left(T^{2}\left(S^{2} T^{2}\right)^{n-1}\right) x\right) \\
& \qquad \begin{array}{l}
{\left[d\left(T^{2}\left(S^{2} T^{2}\right)^{n-1} x, S^{2} T^{2}\left(S^{2} T^{2}\right)^{n-1} x\right)\right]^{3}} \\
\left.\leq\left[d\left(S^{2} T^{2}\left(S^{2} T^{2}\right)^{n-1} x, T^{2} S^{2}\left(T^{2}\left(S^{2} T^{2}\right)^{n-1}\right) x\right)\right]^{3}\right\}
\end{array} \\
& \left\{\begin{array}{l}
{\left[d\left(T^{2}\left(S^{2} T^{2}\right)^{n-1} x, S^{2} T^{2}\left(S^{2} T^{2}\right)^{n-1} x\right)\right]^{2}} \\
+\left[d\left(S^{2} T^{2}\left(S^{2} T^{2}\right)^{n-1} x, T^{2} S^{2}\left(T^{2}\left(S^{2} T^{2}\right)^{n-1}\right) x\right)\right]^{2}
\end{array}\right\} \\
& +\beta d\left(T^{2}\left(S^{2} T^{2}\right)^{n-1} x, S^{2} T^{2}\left(S^{2} T^{2}\right)^{n-1} x\right)
\end{aligned}
$$$$
\leq \alpha \frac{\left[d\left(x_{2 n-1}, x_{2 n}\right)\right]^{3}+\left[d\left(x_{2 n}, x_{2 n+1}\right)\right]^{3}}{\left[d\left(x_{2 n-1}, x_{2 n}\right)\right]^{2}+\left[d\left(x_{2 n}, x_{2 n+1}\right)\right]^{2}}+\beta d\left(x_{2 n-1}, x_{2 n}\right)
$$

$$
\begin{gathered}
\leq \alpha\left[d\left(x_{2 n-1}, x_{2 n}\right)+d\left(x_{2 n}, x_{2 n+1}\right)\right]+\beta d\left(x_{2 n-1}, x_{2 n}\right) \\
\leq(\alpha+\beta) d\left(x_{2 n-1}, x_{2 n}\right)+\alpha d\left(x_{2 n}, x_{2 n+1}\right) \\
(1-\alpha) d\left(x_{2 n}, x_{2 n+1}\right) \leq(\alpha+\beta) d\left(x_{2 n-1}, x_{2 n}\right) \\
d\left(x_{2 n}, x_{2 n+1}\right) \leq \frac{(\alpha+\beta)}{(1-\alpha)} d\left(x_{2 n-1}, x_{2 n}\right) \\
\leq k d\left(x_{2 n-1}, x_{2 n}\right),
\end{gathered}
$$

where $k=\frac{(\alpha+\beta)}{(1-\alpha)}$.

Proceeding in the same manner

$$
d\left(x_{2 n}, x_{2 n+1}\right) \leq k^{2 n-1} d\left(x_{1}, x_{2}\right) .
$$

Also $d\left(x_{n}, x_{m}\right) \leq \sum_{i=n}^{m} d\left(x_{i}, x_{i+1}\right)$ for $m>n$.

Since $k<1$, it follows that the sequence $\left\{x_{n}\right\}$ is Cauchy sequence in the complete metric space $\mathrm{X}$ and so it has a limit in $\mathrm{X}$, that is

$\lim _{n \rightarrow \infty} x_{2 n}=u=\lim _{n \rightarrow \infty} x_{2 n+1}$

and since $\mathrm{T}$ is continuous, we have

$u=\lim _{n \rightarrow \infty} x_{2 n+1}=\lim _{n \rightarrow \infty} T^{2}\left(x_{2 n}\right)=T^{2} u$.

Further,

$$
\begin{aligned}
d\left(x_{2 n+1}, S^{2} u\right) & =d\left(T^{2}\left(S^{2} T^{2}\right)^{n+1} x, S^{2} u\right) \\
& =d\left(T^{2}\left(S^{2} T^{2}\right)^{n+1} x, S^{2}\left(T^{2} u\right)\right)
\end{aligned}
$$

for $u=T^{2} u$

$$
\begin{aligned}
& \leq \frac{\left[d\left(T^{2} u, S^{2} T^{2} u\right)\right]^{3}+\left[d\left(\left(S^{2} T^{2}\right)^{n+1} x, T^{2}\left(S^{2} T^{2}\right)^{n+1} x\right)\right]^{3}}{\left[d\left(T^{2} u, S^{2} T^{2} u\right)\right]^{2}+\left[d\left(\left(S^{2} T^{2}\right)^{n+1} x, T^{2}\left(S^{2} T^{2}\right)^{n+1} x\right)\right]^{2}} \\
& \quad+\beta d\left(T^{2} u,\left(S^{2} T^{2}\right)^{n+1} x\right) \\
& \leq \alpha\left[d\left(T^{2} u, S^{2} T^{2} u\right)+d\left(x_{2 n+2}, x_{2 n+3}\right)\right]+\beta d\left(x_{2 n+2}, T^{2} u\right) \\
& \leq \alpha\left[d\left(u, S^{2} u\right)+d\left(x_{2 n+2}, x_{2 n+3}\right)\right]+\beta d\left(x_{2 n+2}, u\right)
\end{aligned}
$$


Taking limit as $n \rightarrow \infty$, it follows that

$$
d\left(u, S^{2} u\right) \leq 0
$$

which implies

$$
d\left(u, S^{2} u\right)=0 \text { and so } u=S^{2} u=T^{2} u \text {. }
$$

Now consider weak** commutativity of pair $\{S, T\}$ implies that $S^{2} T^{2} u=T^{2} S^{2} u, S^{2} T u=T S^{2} u, S T^{2} u=T^{2} S u$ and so $S^{2} T u=T u$ and $T^{2} S u=S u$.

Now,

$$
\begin{aligned}
& d(u, S u)=d\left(S^{2} T^{2} u, T^{2} S^{2}(S u)\right) \\
& \leq \alpha \frac{\left[d\left(T^{2} u, S^{2} T^{2} u\right)\right]^{3}+\left[d\left(S^{2}(S u), T^{2} S^{2}(S u)\right)\right]^{3}}{\left[d\left(T^{2} u, S^{2} T^{2} u\right)\right]^{2}+\left[d\left(S^{2}(S u), T^{2} S^{2}(S u)\right)\right]^{2}} \\
& +\beta d\left(T^{2} u, S^{2}(S u)\right) \\
& =\alpha \frac{[d(u, u)]+[d(S u, S u)]}{[d(u, u)]+[d(S u, S u)]}+\beta d(u, S u)
\end{aligned}
$$

$(1-\beta) d(u, S u) \leq 0$.

This implies that $(1-\beta) \neq 0$. Hence $d(u, S u)=0$ or $S u=u$.

Similarly, we can show that $T u=u$. Hence, $\mathrm{u}$ is a common fixed point of $\mathrm{S}$ and $\mathrm{T}$.

Now suppose that $\mathrm{v}$ is another common fixed point of $\mathrm{S}$ and $\mathrm{T}$. Then

$$
\begin{aligned}
& d(u, v)=d\left(S^{2} T^{2} u, T^{2} S^{2} v\right) \\
& \leq \alpha \frac{\left[d\left(T^{2} u, S^{2} T^{2} u\right)\right]^{3}+\left[d\left(S^{2} v, T^{2} S^{2} v\right)\right]^{3}}{\left[d\left(T^{2} u, S^{2} T^{2} u\right)\right]^{2}+\left[d\left(S^{2} v, T^{2} S^{2} v\right)\right]^{2}} \\
& +\beta d\left(T^{2} u, S^{2} v\right) \\
& \leq \alpha[d(u, u)+d(v, v)]+\beta d(u, v) \\
& (1-\beta) d(u, v) \leq 0 .
\end{aligned}
$$

Since, $(1-\beta) \neq 0$, then $d(u, v)=0$.
Thus, it follows that $u=v$.Hence $S$ and $T$ have a unique common fixed point.

\section{REFERENCES}

[1]. Banach, S.Sur les operations dans les ensembles abstraits et leur application aux equations integrals, Fund. Math. 3(1922), 133-181.

[2]. Ciric, Lj. B.A generalization of Banach contraction principle, Proc. Amer. Math. Soc. 45(1974), 267-273.

[3]. Fisher, B.Common fixed point and constant mappings on metric spaces, Math. Sem. Notes, Kobe University 5(1977), 319-326.

[4]. Jungck, G.Commuting mappings and fixed points, Amer. Math. Monthly 83(1976), 261-263.

[5]. Lohani, P. C. And Badshah, V. H. Common fixed point and weak**commuting mappings. Bull. Cal. Math. Soc. 87(1995), 289-294.

[6]. Pathak, H. K.Weak* commuting mappings and fixed points. Indian J. Pure Appl. Math. 17(1986), 201-211.

[7]. Ranganathan, S.A fixed point theorem for commuting mapping. Math. Sem. Notes. Kobe. Univ. 6(1978), 351-357.

[8]. Sessa, S.On a weak commutativity condition in fixed point considerations, Publ. Inst. Math. 32(1982), 149-153.

[9]. Singh, S. L.On common fixed point of commuting mappings. Math. Sem. Notes. Kobe. Univ. 5(1977), 131-134. 